\title{
MANAGING PRICE AND SERVICE RATE IN CUSTOMER- INTENSIVE SERVICES UNDER SOCIAL INTERACTIONS
}

\author{
Chengzhang $\mathrm{LI}^{1^{*}}$, Minghui JIANG ${ }^{2}$, Xuchuan YUAN ${ }^{3}$ \\ ${ }^{1}$ School of Management, Harbin Institute of Technology, Harbin City, China \\ ${ }^{2}$ School of Management, Harbin Institute of Technology, Harbin City, China \\ ${ }^{3}$ School of Business, Singapore University of Social Sciences, Singapore
}

Received 20 December 2018; accepted 14 May 2019

\begin{abstract}
This paper investigates the price and service rate decisions in a customer-intensive service in an $\mathrm{M} / \mathrm{M} / 1$ queue system under the influence of social interactions, where a higher value of the service is perceived if more customers purchase the service. The customer-intensive nature of the service requires a low service speed to maintain its quality, which may increase the congestion of the system. Two cases where customers are either homogeneous or heterogeneous in terms of the customer intensity are considered. It is found that social interactions can always benefit the service provider as more expected revenue can be achieved, and potential profits would be lost if the influence of social interations is ignored. For the case with heterogeneous customers, the optimal price and service rate decisions are solved with or without considering social interaction effect. The study finds the proportions of high and low sensitive customers and the social interaction intensity are critical to the operational decisions and the market coverage strategies. These results offer a better understanding on the interplay between the quality-speed conundrum and the influence of social interactions in customers' purchase behaviour in managing customer-intensive services.
\end{abstract}

Keywords: customer-intensive service, M/M/1 queue, heterogeneous customers, price, service rate, social interactions.

JEL Classification: C01, L11, L12, M11.

\section{Introduction}

A customer-intensive service requires a high level of customer contact between the service provider and customers during the delivery and/or consumption process. Examples of customer-intensive services have been given in Anand, Paç, and Veeraraghavan (2011), for example, legal and financial consulting and healthcare services, etc. For these services, a typical characteristic is that the quality perceived by customers is closely related to the service speed; specificaly, the faster service speed, the lower service quality. As the service speed is deter-

${ }^{\star}$ Corresponding author. E-mail: lcz_grown@163.com 
mined by the service rate, service providers of customer-intensive services would prefer a low service rate to reduce the service speed. However, a low service rate limits the service capacity and efficiency, which may also lead to a long queue where customers have to join and suffer from a high waiting cost for consuming the service. Although joining queues incurs waiting costs, customers seem to be more inclined to join long queues in service consumption or buy popular and hot products. Plenty of evidences have shown that customers' consumption decisions are not only determined by the intrinsic value of the service, but also influenced by many other factors which affect their value perception. For example, Spathis, Petridou, and Glaveli (2004) investigate how gender differences affect a customer's perceived service quality in the banking industry. Marand, Tang, and Li (2019) address the impact of deliveryreliability level on customers' value perception and equilibrium behaviours. Nie, Wang, and Yang (2019) find that service providers' maintenance commitments have significant impact on consumers' purchase decisions. Following Yuan and Hwarng (2018), this paper focuses on the influence of social interactions in customers' decision-making process. The phenomenon that customers prefer to buy popular and hot products has been widely observed in the consumer market and extensively documented in the literature. For many customer-intensive services, there also exist similar behavioral patterns in consumers' purchase decisions, i.e., consumers prefer longer queues in service consumption. More discussions on social interactions are provided in the literature review section.

Thus, besides the quality-speed conundrum, service providers of customer-intenstive services may be more unwilling to increase the service rate to reduce the length of the queue and/or the waiting time when customers are influenced by social interactions in service consumption. These two effects imply that the providers of customer-intensive services could benefit more from longer service durations and waiting queues by using lower service rates in operations. The critical tradeoff which needs to be balanced is the increased waiting cost that deters customers to join the queues for service. Besides the service rate decision, service providers also need to consider the price decision in the operations of customer-intensive services. What should be the appropriate service rate and price decisions are critical to the service providers of customer-intensive services to achieve profit optimization.

This paper considers the service rate and price decisions in a customer-intensive service. In contrast to the existing studies on the operational decisions in this type of service, it takes the influence of social interactions into consideration in customers' purchase decision-making. In particular, it considers a single service firm with the objective to maximize the revenue which provides a customer-intensive service in the consumer market. Following Yuan and Hwarng (2018), the influence of social interactions is characterized by the phenomenon that customers perceive a higher value if more customers purchase the same service. Besides, the paper also considers customers' heterogeneity in terms of the customer intensity. The service provider is operated as an $\mathrm{M} / \mathrm{M} / 1$ queueing system to capture the congestion effect due to service rate constraint. The service provider can expand the market coverage to serve more customers by using a higher service rate or a lower price. However, due to the customer-intensive nature of the service, a higher service rate reduces the service quality which may drive customers away. The service provider has to make tradeoffs between service speed and quality under the situation that customers' purchase decisions are influenced by social interactions. 
This paper is organized as follows. Section 1 provides the literature review on the operations management in customer-intensive services and the influence of social interactions on customers' purchase decision-making. Section 2 describes the model. Section 3 analyses the optimal price and service rate decisions with homogeneous customers with/without the influence of social interactions. Section 4 characterizes the optimal decisions with heterogeneous customers. The last section concludes the paper.

\section{Related studies}

This paper mainly reviews two streams of literature that are related to the study: the operational decisions in customer-intensive services and the influence of social interactions on customers' purchase decision-making.

\subsection{Operational decisions in customer-intensive services}

There are extensive studies focusing on service operations management; see, e.g., Stidham (2002), Ata and Shneorson (2006), and Hassin and Haviv (2003). As one important category of common used services, customer-intensive services have been studied by many scholars. Anand et al. (2011) demonstrate that the customer intensity plays an important role in the service operational decisions and leads to outcomes very different from traditional service operations. Li, Guo, and Lian (2016) consider a service system for a customer-intensive service in which customers are boundedly rational and make their queue joining decisions according to a logit model. They demonstrate that the revenue function is unimodal in terms of the service rate and other decision variables and find the socially optimal price is always lower than the individual level in competition. X. Li, Q. Li, Guo, and Lian (2017) extend the service-speed competition game with boundedly rational customers to the case with general reward functions and multiple customer-intensive service providers. They find that the competition between two service providers benefits the customers which improves their utilities while more customers can be served. Based on the work in Anand et al. (2011), Ni et al. (2013) consider two classes of customers into consideration in terms of customer intensity. Their research indicates that under some mild conditions, the provider could gain more revenue by treating different classes seperately than by adopting a uniform treatment for both types of customers. Li, Jiang, and Yuan (2018) investigate the optimal price and service rate decisions in the operations of customer-intensive services, where customers are assumed to be forward-looking in purchase decision-making and heterogeneous in their reservation utilities. They aim to study the impact of customers' forward-looking behavior and their heterogeneity on the operational decisions in customer-intensive services.

Motivating by the above studies, where assuming there are two types of customers in a customer-intensive service market, namely high sensitive customers and low sensitive customers. High sensitive customers are susceptible to the service speed while low sensitive customers are less susceptible in quality perception. Different from those existing studies, in additional to the quality-speed conundrum, it also considers the influence of social interactions in customers' purchase decision-making in the context of the customer-intensive service. 


\subsection{Influence of social interactions on customers' purchase behavior}

The influence of social interactions on customers' purchase behavior has been studied extensively in various domains by both the empirical and theoretical approaches. Existing studies have demonstrated that social interactions can influence customers' preferences and purchase decisions through various channels (Hanson \& Putler, 1996; Onnela, Reed-Tsochas \& Stanley, 2010; Simonsohn \& Ariely, 2008). Bohlmann, Rosa, Bolton, and Qualls (2006) find social interactions can change customer's satisfaction judgment. The influence of social interactions on customers' utlity and value perception can be substantial. Veeraraghavan and Debo (2009) find social interactions make customers choose longer queues which include more customers waiting for the service. Hartmann (2010) finds that 35\% of customers' utility is attributed to the influence of social interactions. Moretti (2011) argues that nearly $32 \%$ of sales in a cinema are contributed to social interactions. There are many studies focusing on the price, service speed and service quality decisions considering the influence of social interactions. Godes (2016) focuses the product quality decision when consumers are more likely to exchange information through word-of-mouth communication, he finds that the optimal quality path may either increase or decrease as word-of-mouth expands. Yu, Debo, and Kapuscinski (2016) study the impact of consumers' reviews on a company's dynamic pricing strategy in the presence of strategic consumers. They find consumer surplus can decrease due to the consumer-generated quality information. Qiu and Whinston (2017) focus on the optimal operational strategies of a monopolistic firm when consumers share purchase decisions which enables behavioral observational learning in social networks. Papanastasiou and Savva (2017) finds that the presence of social learning has significant structural implications for optimal pricing policies with forward-looking consumers using a simple two-period model. Similar dynamic pricing problem is discussed in Campbell (2015), where the product information is diffusing through word of mouth. Zhang, Liu, and Chen (2015) study the impact of observational learning in networks of friends versus strangers on consumers' purchase decisions, where a consumer makes purchase decision after receiving a private signal about product quality and observing the actions of others. One of the extensions in our paper is also related to V. Kostami, D. Kostamis, and Ziya (2017), where customers are heterogeneous in terms of their willingness to pay for the service, and their utilities are affected by other customers in the system.

From the above literature review, there exists gaps in the existing studies on the operational decisions, particularly the price and service rate, in customer-intensive services, where the impact of social interactions on customers' purchase behavior has not been considered. $\mathrm{Ni}$ et al. (2013) extend the work of Anand et al. (2011) and indicates that under some mild conditions, the provider could gain more revenue by treating different classes seperately than by adopting a uniform treatment for both types of customers. But they do not explore the different optimal operational strategies for the service provider considering different classes of customers, which is finished in our work. Following the framework in Brock and Durlauf (2001, 2007), similar to Yuan and Hwarng (2018), this paper models the influence of social interactions on customers' purchase decisions in a way that the more customers who purchase the service, the higher value of the service is perceived. There could be many underlying forces that drive the social interaction influence, e.g., the reference group effect and the network externalities, which are not the focus in this paper. Instead of identifying the causes 
and measuring the influence of social interactions, it takes this social interaction influence directly into consumers' purchase decision-making on the basis of its prevalent existence. To the best of our knowledge, our study fills the gap of current literature and provides better understanding on the influence of social interactions in managing operations in customerintensive services.

\section{Model setup}

Considering a revenue maximizing service provider (thereafter, the firm and the service provider are used interchangeably) which offers a customer-intensive service to customers in the market. The service is delivered through an $\mathrm{M} / \mathrm{M} / 1$ queue where the capacity, measured by its service rate, determines the service speed. Besides the service rate, the firm also has to make the price decision. Due to the random arrivals of customers and the variated service time, a queue exists and customers have to join the queue to procure the service. In the following section, customers' purchase decision and queue joining decision are used interchangeably. The firm can speed up the service with a higher service rate to shorten the service time and the waiting time. However, the service quality will decline due to the fast service speed for this customer-intensive service. The potential demand for the customer-intensive service, denoted as $\Lambda$, is fixed, and the customers' purchase decisions are affected by social interactions. In terms of customers' queue joining decisions, following Li et al. (2018), this study considers customers are forward-looking, i.e., if customers' expected value is larger than their reservation value, they will join the queue to procure the service; otherwise, they will not join the queue. Key notations used in this paper are listed in Table 1.

\subsection{Customer's purchase decision-making}

Firstly, the study considers how potential customers make the purchase decisions on this customer-intensive service taking into account the influence of social interactions in their value perception. As discussed in Anand et al. (2011), without considering other factors, the value of a customer-intensive service is given as below:

$$
V(\mu)=\left(V_{b}+\alpha \mu_{b}-\alpha \mu\right)^{+}
$$

where the customer-intensity $\alpha>0$.

Table 1. Notations and definitions

\begin{tabular}{|c|l|c|l|}
\hline Notations & \multicolumn{1}{|c|}{ Definition } & Notations & \multicolumn{1}{|c|}{ Definition } \\
\hline$\Lambda$ & Potential arrival rate (demand) & $u$ & Expected value \\
\hline$\mu$ & Service rate (capacity) & $U_{b}^{r}$ & Reservation value \\
\hline$\mu_{b}$ & Base service rate & $p$ & Service Price \\
\hline$\lambda$ & Effective arrival rate (demand) & $\lambda(\mu, p)$ & Equilibrium arrival rate (demand) \\
\hline$\delta$ & Social interaction intensity & $c$ & Waiting cost per unit of time \\
\hline$\alpha$ & Customer intensity & $\alpha^{H}$ & High sensitive customer intensity \\
\hline$W(\mu, \lambda)$ & Expected waiting time & $\alpha^{L}$ & Low sensitive customer intensity \\
\hline$V_{b}$ & Intrinsic value of the service & $V$ & Perceived service value \\
\hline$v_{2}(\lambda)$ & Social value & $v_{1}\left(\mu, \mu_{b}\right)$ & Customer-intensive service value \\
\hline
\end{tabular}


Based on the above value function for the customer-intensive service, in the model, due to social interactions, it assumes customers' perceived service value, denoted as $V$, consists of two parts, namely the customer-intensive service value and social value. Specifically, it assumes the customer-intensive service value is given as $V(\mu)=V_{b}+\alpha \mu_{b}-\alpha \mu$, which is the sum of the intrinsic value of the service, $V_{b}$, and the service quality value due to the customer-intensive nature of the service, i.e., $v_{1}\left(\mu, \mu_{b}\right)=\alpha\left(\mu_{b}-\mu\right)$. If $\mu \leq \mu_{b}$, customers perceive a positive service quality value, i.e., $v_{1}\left(\mu, \mu_{b}\right) \geq 0$; if $\mu>\mu_{b}$, then the service quality value will be negative.

Following Yuan and Hwarng (2018), the social value due to the influence of social interactions is assumed to be $v_{2}(\lambda)=\delta \lambda$, where $\delta>0$ is denoted as the social interaction intensity. Our model of the social value is on the basis of the definition given by Brock and Durlauf (2001), where social interactions capture the fact that "the utility or payoff an individual receives from a given action depends directly on the choices of others in that individual's reference group". Note that using the linear form for analytical simplicity. The managerial implications qualitatively hold for other forms as long as the social value increases in the demand rate. The resulting perceived service value for the customer-intensive service is expressed as:

$$
V=V_{b}+\alpha \mu_{b}-\alpha \mu+\delta \lambda, \text { where } \alpha>0, \delta>0 .
$$

For a potential customer, the net service value is calculated as below:

$$
u(\mu, \lambda, p)=V_{b}+\alpha\left(\mu_{b}-\mu\right)+\delta \lambda-p-c W(\mu, \lambda),
$$

where $W(\mu, \lambda)$ is the expected waiting time.

Since customers are forward-looking, the reservation value measures the least value that customers require from the service consumption. For simplicity, it is assumed every customer has the same reservation value $U_{b}^{r}$. Given the service rate and price, there are three types of outcomes: 1) $u(\mu, \Lambda, p)>U_{b}^{r}$, i.e., all potential customers will join the queue; 2) If $\max _{\lambda \in[0, \Lambda]} u(\mu, \lambda, p)<U_{b}^{r}$, i.e., nobody will join the queue; 3$) \exists \lambda \in[0, \Lambda], u(\mu, \lambda, p)=U_{b}^{r}$, i.e., in equilibrium, customers' expected value equals the reservation value. As the equilibrium arrival rate depends on the price and service speed, the equilibrium arrival rate is denoted as $\lambda(\mu, p)$.

\subsection{Service provider's operational decisions}

The objective of the service provider is to maximize the revenue by adjusting the service rate freely and the price through the following revenue optimization model

$$
\max _{\mu, p} R(\mu, p)=\max _{\mu, p} p \lambda(\mu, p) .
$$

Given the service rate and the arrival rate, customers' expected waiting time in an $\mathrm{M} / \mathrm{M} / 1$ queue system is given as follows:

$$
W(\mu, \lambda)=\left\{\begin{array}{c}
\frac{1}{\mu-\lambda}, \text { if } 0 \leq \lambda \leq \mu, \\
\infty, \quad \text { otherwise }
\end{array}\right.
$$


Combining customers' queue joining decision, the optimal service rate and price decisions can be solved by (1).

As the parameter $\alpha$ captures the sensitivity on the value of the service due to the impact of the service speed, customers can be homogeneous or heterogeneous according to different classes of customer intensity. In Section 4, the study focuses on homogeneous customers who have the same customer intensity in value perception. In Section 5, it makes further research on heterogeneous customers who share different levels of customer intensity when perceiving the service quality value.

\section{Service operations with homogeneous customers}

This section studies on homogeneous customers who share the same customer intensity in value perception. Assuming $V_{b}-c / \mu_{b}-U_{b}^{r} \geq 0$ in the following sections. This condition guarantees that some customers will join the queue even if the firm uses the base service rate. This assumption ensures that the service provider can always achieve positive revenues through appropriate operational decisions, i.e., price and service rate.

\subsection{Price and service rate decisions with social interactions}

Under the influence of social interactions, the optimal price and service rate decisions are given by the following result:

Proposition 1. The optimal service rate and price decisions are given as:

$$
\mu_{m}=\lambda_{m}+\sqrt{c / \alpha}, p_{m}=V_{b}+\alpha\left(\mu_{b}-\lambda_{m}\right)+\delta \lambda_{m}-2 \sqrt{c \alpha}-U_{b}^{r},
$$

and the optimal revenue is given by $R_{m}=\left(V_{b}+\alpha \mu_{b}+(\delta-\alpha) \lambda_{m}-2 \sqrt{c \alpha}-U_{b}^{r}\right) \lambda_{m}$, where

$$
\lambda_{m}= \begin{cases}\min \left(\frac{V_{b}+\alpha \mu_{b}-2 \sqrt{c \alpha}-U_{b}^{r}}{2(\alpha-\delta)}, \Lambda\right), & \delta<\alpha, \\ \Lambda, & \delta \geq \alpha .\end{cases}
$$

By Proposition 1, it can be seen that social interactions have an important effect on the service provider's operational decision-making. If the social interaction is strong enough, namely $\delta \geq \alpha$, it is beneficial for the service provider to cover the whole market, i.e., $\lambda_{m}=\Lambda$ ; while if $\delta<\alpha$, it is not always the best choice to cover the whole market. The service provider should take all the other factors into consideration such as the intrinsic service value, the customer intensity, the base service value, the social interaction intensity, the reservation utility, and the waiting cost per unit time, when making the market coverage decision.

By Proposition 1, it can be seen the optimal service rate decreases in the customer intensity while increases in the waiting cost per unit time $c$, which implies that when customers are more averse in waiting, the service provider should use a high service rate or charge a lower price; under the partial market coverage case, the optimal service rate and price increase in the base service rate $\mu_{b}$ and decrease in the reservation value $U_{b}^{r}$; therefore, if customers have a higher reservation value before making purchase decisions, the service provider make 
a lower price or use a lower service rate. For the market with partial coverage, the optimal service rate increases in the social interaction intensity $\delta$, while the optimal price is not influenced by the social interaction intensity. In contrast, the optimal price is increasing in $\delta$ and the optimal service speed is not directly determined by social interaction intensity if the market is fully covered.

Numerical studies are conducted to illustrate the most appropriate price, service rate and revenues according to different $\alpha$ and $\delta$ separately. In Figure 1, the parameter values used in the numerical example are $\Lambda=5, V_{b}=6, c=1, \mu_{b}=8, \delta=1, U_{b}^{r}=1$.

In Figure 2, the parameter values used in the numerical example are $\Lambda=5, V_{b}=6, c=1$, $\mu_{b}=8, \alpha=2, U_{b}^{r}=1$.
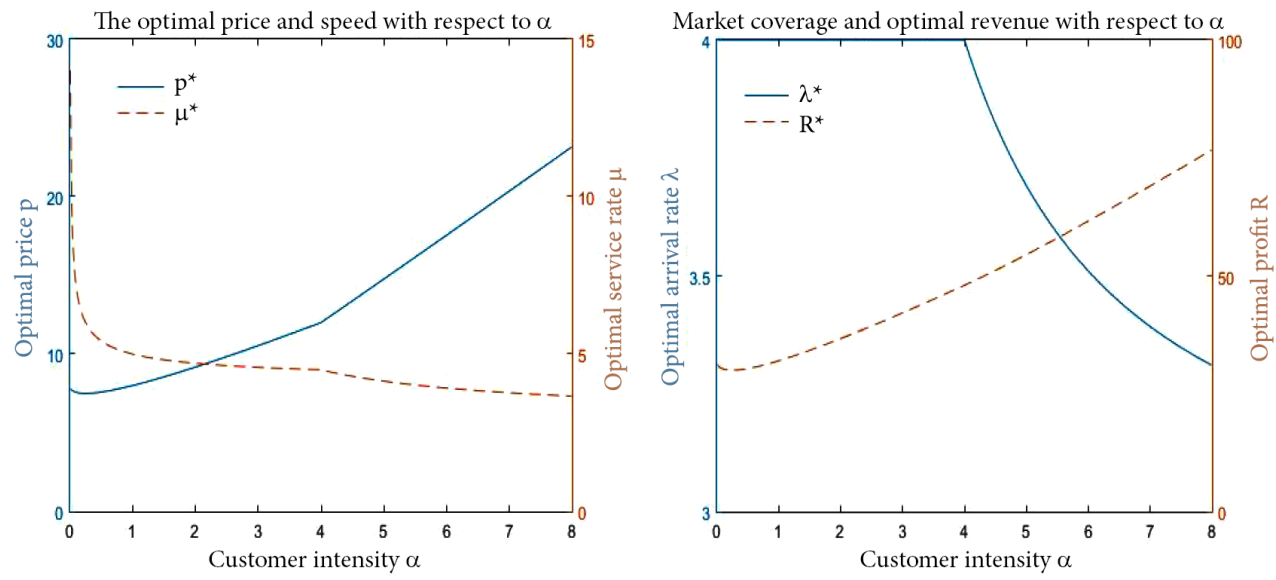

Figure 1. The left panel illustrates the optimal service rate and price with respect to the customer intensity $\alpha$. The right panel illustrates the arrival rate in equilibrium and the optimal revenue with respect to $\alpha$
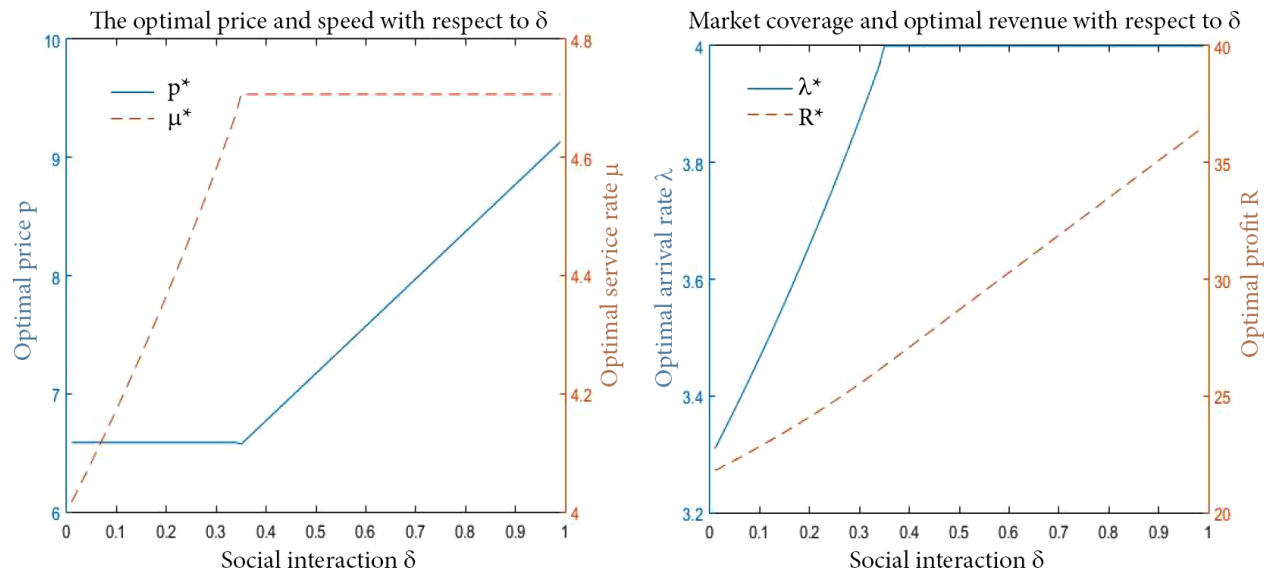

Figure 2. The left panel illustrates the optimal service rate and price with respect to the social interaction intensity $\delta$. The right panel illustrates the arrival rate in equilibrium and the optimal revenue with respect to $\delta$ 
By Figure 2, It can be seen that social interactions can benefit the customer-intensive service provider. With the increase of social interaction intensity, a larger market could be covered while a higher service rate can be used, where the firm can charge a higher price and always gain more revenue.

\subsection{Comparisons between the cases with and without social interactions}

This section first considers the case where the service provider ignores the influence of social interactions in operational decision-making, and then compare the cases with and without taking social interactions into consideration in customers' value perception. If the service provider ignores the influence of social interactions, following Li et al. (2018), as in equilibrium, customers' expected value and their reservation value are equal, the price satisfies $p=V_{b}+\alpha\left(\mu_{b}-\mu\right)-c W(\mu, \lambda)-U_{b}^{r}$. After substituting it into the revenue function, the service rate is solved given the arrival rate, and then solve the optimal service rate after finding the optimal arrival rate. Mathematically, the optimal service rate without social interaction effect can be solved by the following problem

$$
\max _{\lambda \in[0, \Lambda], \lambda<\mu}\left(V_{b}+\alpha\left(\mu_{b}-\mu\right)-\frac{c}{\mu-\lambda}-U_{b}^{r}\right) \lambda .
$$

To solve (3), for $\lambda \in[0, \Lambda]$, namely given the market coverage choice, the revenue function (3) is concave in $\mu$, and the optimal service rate satisfies $\mu(\lambda)=\lambda+\sqrt{c / \alpha}$, i.e., the service provider uses the optimal service rate given the arrival rate. Depending on whether the market is partially covered or fully covered, there are following result when social interactions are ignored:

Proposition 2: If the market is covered partially, the equlibrium arrival rate, the optimal decisions, and the revenue can be expressed as below:

$$
\begin{gathered}
\lambda^{*}=\left(V_{b}+\alpha \mu_{b}-2 \sqrt{c \alpha}-U_{b}^{r}\right) /(2 \alpha), \mu^{*}=\left(V_{b}+\alpha \mu_{b}-U_{b}^{r}\right) / 2 \alpha, \\
p^{*}=\left(V_{b}+\alpha \mu_{b}-2 \sqrt{c \alpha}-U_{b}^{r}\right) / 2, R^{*}=\left(V_{b}+\alpha \mu_{b}-2 \sqrt{c \alpha}-U_{b}^{r}\right)^{2} / 4 \alpha ;
\end{gathered}
$$

In the market with full coverage, the optimal demand is $\lambda^{*}=\Lambda$, the best service rate and price are $\mu^{*}=\Lambda+\sqrt{c / \alpha}, p^{*}=V_{b}+\alpha\left(\mu_{b}-\Lambda\right)-2 \sqrt{c \alpha}-U_{b}^{r}$, respectively. The maximum revenue is $R^{*}=\left(V_{b}+\alpha\left(\mu_{b}-\Lambda\right)-2 \sqrt{c \alpha}-U_{b}^{r}\right) \Lambda$.

By Propositions 1 and 2, numerical studies are conducted to compare the cases with and without considering social interactions. Figure 3 illustrates the optimal prices and revenues under two cases. In the numerical example, the parameter values are $\Lambda=5, V_{b}=6, c=1$, $\mu_{b}=8, U_{b}^{r}=1, \delta=1$. In Figure 3, the subscript $m$ refers to the case with social interactions, and the subscript $n$ refers to the case without social interactions.

As can be seen from Figure 3, under the influence of social interactions, the firm could charge a higher price in the market with full coverage compared to the case without social interactions; while the optimal prices are the same when the market is partially covered. It can also be observed that the optimal revenue under social interactions is always higher than that without social interactions. Thus, under the influence of social interactions on custom- 

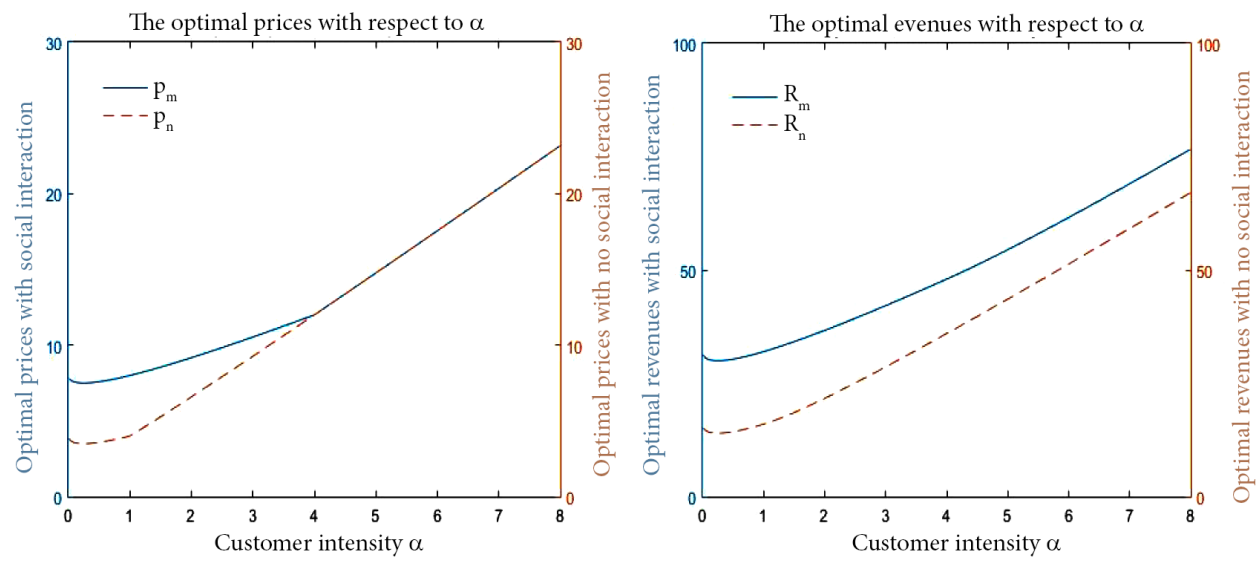

Figure 3. The left panel illustrates the optimal prices with respect to $\alpha$ with and without social interaction effect. The right panel illustrates the optimal revenues in equilibrium with respect to $\alpha$ with and without social interation effect

ers' value perception, the firm in customer-intensive service market will gain more demand and can charge a higher price, which surely achieves more revenue. The result also implies that substantial profits will be lost if the social interaction effect is ignored in operational decision-making.

\section{Service operations with heterogeneous customers}

\subsection{Price and service rate decisions without social interactions}

This section focuses on the situation with heterogeneous customers in terms of customer intensity, where customers are divided into different groups according to their sensitivities to the service rate in value perception. For analytical simplicity, assuming there are two types of customers for the customer-intensive service, namely high sensitive customers and low sensitive customers. High sensitive customers are susceptible to the service speed while low sensitive customers are less susceptible. In the model, using $\alpha^{H}$ and $\alpha^{L}$ to measure the high sensitive and low sensitive customers' value perceptions on the customer-intensive service due to the impact of the service rate, respectively, with $\alpha^{H}>\alpha^{L}>0$. The proportions of high sensitive and low sensitive customers are $q \in[0,1]$ and $1-q$ in the market, respectively.

The arrival rate of the high sensitive customers is denoted as $\lambda_{1} \in[0, q \Lambda]$, and the arrival rate of the low sensitive customers as $\lambda_{2} \in[0,(1-q) \Lambda]$. The total arrival rate for the service is $\lambda=\lambda_{1}+\lambda_{2}$. The objective of the service provider is to choose the service rate and price to maximize the expected revenue.

In equilibrium, the service provider should adjust the service rate and price to make the expected value of at least one of the two types of customers equal to their reservation value. Otherwise, the firm can use a higher price to get more revenues without affecting the effective arrival rate. This section first considers two operational strategies, I and II. The strategy I refers to the case where the service provider reduces the high sensitive customers' expected 
value to their reservation value in equilibrium. While the strategy II is to reduce the low sensitive customers' expected value to their reservation value in equilibrium. The optimal equilibrium service rate and price under these two operational strategies are denoted as $\mu_{\mathrm{I}}$ and $p_{\mathrm{I}}, \mu_{\mathrm{II}}$ and $p_{\mathrm{II}}$, respectively; the maximum revenues under the two operational strategies are denoted as $R_{\mathrm{I}}$ and $R_{\mathrm{II}}$, respectively; the optimal effective arrival rate under the two operational strategies are denoted as $\lambda_{\mathrm{I}}$ and $\lambda_{\mathrm{II}}$, respectively.

As discussed in Section 3, the service quality value $v_{1}\left(\mu, \mu_{b}\right)=\alpha\left(\mu_{b}-\mu\right)$ is determined by the selected service rate and the base service rate. Particularly, if $\mu<\mu_{b}$, high sensitive customers can always get more service values than low sensitive ones; while if $\mu \geq \mu_{b}$, low sensitive customers are more likely to purchase the service. Based on this observation, the study considers the cases with $\mu<\mu_{b}$ and $\mu \geq \mu_{b}$, separately.

(1) The case with $\mu<\mu_{b}$

For the case with $\mu<\mu_{b}$, the service quality value $v_{1}\left(\mu, \mu_{b}\right)=\alpha\left(\mu_{b}-\mu\right)$ will be positive. For the same service rate, there is relationship $\alpha^{H}\left(\mu_{b}-\mu\right)>\alpha^{L}\left(\mu_{b}-\mu\right)$, which means high sensitive customers can always get larger values than low sensitive ones. The research firstly considers the strategy I. If the service provider adopts strategy I, i.e., the service provider reduces high sensitive customers' expected value to their reservation level in equilibrium. This decision can hold in equilibrium, since high sensitive customers' expected value is same as the reservation value; while low sensitive customers' utility is lower than the reservation value. So high sensitive customers will choose the service while low sensitive customers will not be attracted. Based on the proof of Proposition 2, the optimal decisions and revenue under the strategy I can be expressed as below:

$$
\begin{gathered}
\lambda_{\mathrm{I}}=\min \left(\frac{V_{b}+\alpha^{H} \mu_{b}-2 \sqrt{c \alpha^{H}}-U_{b}^{r}}{2 \alpha^{H}}, q \Lambda\right), \mu_{\mathrm{I}}=\lambda_{\mathrm{I}}+\sqrt{c / \alpha^{H}}, \\
p_{\mathrm{I}}=V_{b}+\alpha^{H}\left(\mu_{b}-\lambda_{\mathrm{I}}\right)-2 \sqrt{c \alpha^{H}}-U_{b}^{r}, R_{\mathrm{I}}=\left(V_{b}+\alpha^{H}\left(\mu_{b}-\lambda_{\mathrm{I}}\right)-2 \sqrt{c \alpha^{H}}-U_{b}^{r}\right) \lambda_{\mathrm{I}} .
\end{gathered}
$$

If the service provider adopts strategy II to reduce low sensitive customers' expected value to their reservation value in equilibrium, then all the high sensitive customers will choose the service since for those customers, their expected value is higher than the reservation value. So the optimal decisions and revenue under strategy II are given as follows:

$$
\begin{gathered}
\lambda_{\mathrm{II}}=\max \left(\frac{V_{b}+\alpha^{L} \mu_{b}-2 \sqrt{c \alpha^{L}}-U_{b}^{r}}{2 \alpha^{L}}, q \Lambda\right), \mu_{\mathrm{II}}=\lambda_{\mathrm{II}}+\sqrt{c / \alpha^{L}}, \\
p_{\mathrm{II}}=V_{b}+\alpha^{L}\left(\mu_{b}-\lambda_{\mathrm{II}}\right)-2 \sqrt{c \alpha^{L}}-U_{b}^{r}, R_{\mathrm{II}}=\left(V_{b}+\alpha^{L}\left(\mu_{b}-\lambda_{\mathrm{II}}\right)-2 \sqrt{c \alpha^{L}}-U_{b}^{r}\right) \lambda_{\mathrm{II}} .
\end{gathered}
$$

Based on the above two strategies, it can be concluded that the proportions of these two types of customers play an important role in the service provider's operational decision-making. The service provider can choose different operational strategy to maximize the expected revenue based on the actual values of $q, \alpha^{H}$ and $\alpha^{L}$. If $R_{\mathrm{I}}>R_{\mathrm{II}}$, the service provider will prefer strategy I; while strategy II will be adopted when $R_{\mathrm{I}} \leq R_{\mathrm{II}}$. 
(2) The case with $\mu \geq \mu_{b}$

For the case with $\mu \geq \mu_{b}$, the service quality value $v_{1}\left(\mu, \mu_{b}\right)=\alpha\left(\mu_{b}-\mu\right)$ will be negative, and for the same service rate, there is relationship $\alpha^{H}\left(\mu_{b}-\mu\right) \leq \alpha^{L}\left(\mu_{b}-\mu\right)$, which means low sensitive customers can always get larger values than high sensitive ones. In this situation, if the service provider adopts the strategy to reduce high sensitive customers' expected utility to their reservation value in equilibrium, all the low sensitive customers will choose the service since low sensitive customers' value is higher than their reservation value. By Proposition 2, the optimal decisions and revenue under strategy I are given as below:

$$
\begin{gathered}
\lambda_{\mathrm{I}}=\max \left(\frac{V_{b}+\alpha^{H} \mu_{b}-2 \sqrt{c \alpha^{H}}-U_{b}^{r}}{2 \alpha^{H}},(1-q) \Lambda\right), \mu_{\mathrm{I}}=\lambda_{\mathrm{I}}+\sqrt{c / \alpha^{H}}, \\
p_{\mathrm{I}}=V_{b}+\alpha^{H}\left(\mu_{b}-\lambda_{\mathrm{I}}\right)-2 \sqrt{c \alpha^{H}}-U_{b}^{r}, R_{\mathrm{I}}=\left(V_{b}+\alpha^{H}\left(\mu_{b}-\lambda_{\mathrm{I}}\right)-2 \sqrt{c \alpha^{H}}-U_{b}^{r}\right) \lambda_{\mathrm{I}} .
\end{gathered}
$$

For strategy II, the service provider will reduce low sensitive customers' expected value to their reservation value in equilibrium. This strategy can hold in equilibrium, since low sensitive customers' expected value is the same as reservation value, while high sensitive customers' value is lower than reservation value. Therefore, high sensitive customers will not join the queue to purchase the service and the optimal decisions and revenue are given as below:

$$
\begin{gathered}
\lambda_{\mathrm{II}}=\min \left(\frac{V_{b}+\alpha^{L} \mu_{b}-2 \sqrt{c \alpha^{L}}-U_{b}^{r}}{2 \alpha^{L}},(1-q) \Lambda\right), \mu_{\mathrm{II}}=\lambda_{\mathrm{II}}+\sqrt{c / \alpha^{L}}, \\
p_{\mathrm{II}}=V_{b}+\alpha^{L}\left(\mu_{b}-\lambda_{\mathrm{II}}\right)-2 \sqrt{c \alpha^{L}}-U_{b}^{r}, R_{\mathrm{II}}=\left(V_{b}+\alpha^{L}\left(\mu_{b}-\lambda_{\mathrm{II}}\right)-2 \sqrt{c \alpha^{L}}-U_{b}^{r}\right) \lambda_{\mathrm{II}} .
\end{gathered}
$$

Based on the above discussion, the service provider can choose different operational strategy to maximize the revenue depending on the values of $q, \alpha^{H}$ and $\alpha^{L}$. The service provider will choose either strategy I or II based on the comparison of the expected revenue generated by the corresponding operational decisions.

\subsection{Price and service rate decisions with social interactions}

This section considers the case with social interactions. The optimal service rate and price decisions under the influence of social interactions will be more complex than the situation without social interactions. In this section, we solve the optimal operational decisions and study how customer intensity and social interactions affect the optimal decisions in equilibrium. It turns out the magnitudes of the social interaction intensity and the customer intensity play an important role in the firm's operational decisions. Following similar approaches used in Yuan and Hwarng (2018), the cases with $\delta \geq \alpha^{H}, \alpha^{L}<\delta<\alpha^{H}$, and $\delta \leq \alpha^{L}$ are considered separately.

(1) Optimal operational decisions with $\delta \geq \alpha^{H}$

For the case with $\delta \geq \alpha^{H}$, the price and service rate decisions under the two operational strategies are denoted separately. 
- If $\mu_{b} \geq q \Lambda+\sqrt{c / \alpha^{H}}$, the optimal decisions and revenue under the strategy I:

$$
\begin{gathered}
p_{\mathrm{I}}=V_{b}+\alpha^{H}\left(\mu_{b}-q \Lambda\right)+\delta q \Lambda-2 \sqrt{c \alpha^{H}}-U_{b}^{r}, \mu_{\mathrm{I}}=q \Lambda+\sqrt{c / \alpha^{H}}, \\
R_{\mathrm{I}}=\left(V_{b}+\alpha^{H} \mu_{b}+q \Lambda\left(\delta-\alpha^{H}\right)-2 \sqrt{c \alpha^{H}}-U_{b}^{r}\right) q \Lambda ;
\end{gathered}
$$

- If $\mu_{b}<q \Lambda+\sqrt{c / \alpha^{H}}$, the optimal decisions and revenue under the strategy I:

$$
\begin{gathered}
p_{\mathrm{I}}=V_{b}+\alpha^{H}\left(\mu_{b}-\Lambda\right)+\delta \Lambda-2 \sqrt{c \alpha^{H}}-U_{b}^{r}, \mu_{\mathrm{I}}=\Lambda+\sqrt{c / \alpha^{H}} \\
R_{\mathrm{I}}=\left(V_{b}+\alpha^{H} \mu_{b}+\Lambda\left(\delta-\alpha^{H}\right)-2 \sqrt{c \alpha^{H}}-U_{b}^{r}\right) \Lambda
\end{gathered}
$$

- The optimal decisions and revenue under the strategy II:

$$
\begin{gathered}
p_{\mathrm{II}}=V_{b}+\alpha^{L}\left(\mu_{b}-\lambda_{\mathrm{II}}\right)+\lambda_{\mathrm{II}}-2 \sqrt{c \alpha^{L}}-U_{b}^{r}, \mu_{\mathrm{II}}=\lambda_{\mathrm{II}}+\sqrt{c / \alpha^{L}}, \\
R_{\mathrm{II}}=\left(V_{b}+\alpha^{L} \mu_{b}+\lambda_{\mathrm{II}}\left(\delta-\alpha^{L}\right)-2 \sqrt{c \alpha^{L}}-U_{b}^{r}\right) \lambda_{\mathrm{II}},
\end{gathered}
$$

where $\lambda_{\text {II }}=\max \left((1-q) \Lambda, \min \left(\Lambda, \mu_{b}-\sqrt{c / \alpha^{L}}\right)\right)$.

Denoting $p^{*}, \mu^{*}$, and $\lambda^{*}$ as optimal decisions and the equilibrium arrival rate for the service provider. Based on the above two strategies, the service provider's optimal decisions, the market coverage and the revenue are given by the following result (the proof is in the appendix):

Proposition 3. For the case with $\delta \geq \alpha^{H}$, the following cases hold:

- If $\mu_{b} \geq q \Lambda+\sqrt{c / \alpha^{H}}$, and the optimal price and service rate decisions depend on the comparison of the revenues from the two strategies,

- If $R_{\mathrm{I}} \geq R_{\mathrm{II}}$, then $\lambda^{*}=q \Lambda$, and $\mu^{*}=\mu_{\mathrm{I}}, p^{*}=p_{\mathrm{I}}, R^{*}=R_{\mathrm{I}}$;

- If $R_{\mathrm{I}}<R_{\mathrm{II}}$, then $\lambda^{*}=\max \left((1-q) \Lambda, \min \left(\Lambda, \mu_{b}-\sqrt{c / \alpha^{L}}\right)\right)$, and $\mu^{*}=\mu_{\mathrm{II}}, p^{*}=p_{\mathrm{II}}$, $R^{*}=R_{\mathrm{II}}$.

- If $\mu_{b}<q \Lambda+\sqrt{c / \alpha^{H}}$, the optimal price and service rate decisions depend on the comparison of the revenues from the two strategies,

- If $R_{\mathrm{I}} \geq R_{\mathrm{II}}$, then $\lambda^{*}=\Lambda$, and $\mu^{*}=\mu_{\mathrm{I}}, p^{*}=p_{\mathrm{I}}, R^{*}=R_{\mathrm{I}}$;

- If $R_{\mathrm{I}}<R_{\mathrm{II}}$, then $\lambda^{*}=\max \left((1-q) \Lambda, \min \left(\Lambda, \mu_{b}-\sqrt{c / \alpha^{L}}\right)\right)$, and $\mu^{*}=\mu_{\mathrm{II}}, p^{*}=p_{\mathrm{II}}$, $R^{*}=R_{\mathrm{II}}$.

By Proposition 3, to achieve revenue maximization, the service provider can implement the corresponding price and service rate decisions by comparing the expected revenues from strategy I and strategy II.

(2) Optimal strategic decisions with $\alpha^{L}<\delta<\alpha^{H}$

For the case with $\alpha^{L}<\delta<\alpha^{H}$, based on Proposition 1, the optimal arrival rate with $\alpha=\alpha^{H}$ is given as below:

$$
\lambda_{m}^{H}=\min \left(\frac{V_{b}+\alpha^{H} \mu_{b}-2 \sqrt{c \alpha^{H}}-U_{b}^{r}}{2\left(\alpha^{H}-\delta\right)}, \Lambda\right) .
$$


The price and service rate decisions under two operational strategies are denoted as below:

- If $\lambda_{m}^{H}+\sqrt{c / \alpha^{H}}>\mu_{b}$, the decisions and revenue under operational strategy I:

$$
\begin{gathered}
p_{\mathrm{I}}=V_{b}+\alpha^{H}\left(\mu_{b}-\lambda_{\mathrm{I}}\right)+\delta \lambda_{\mathrm{I}}-2 \sqrt{c \alpha^{H}}-U_{b}^{r}, \mu_{\mathrm{I}}=\lambda_{\mathrm{I}}+\sqrt{c / \alpha^{H}}, \\
R_{\mathrm{I}}=\left(V_{b}+\alpha^{H}\left(\mu_{b}-\lambda_{\mathrm{I}}\right)+\delta \lambda_{\mathrm{I}}-2 \sqrt{c \alpha^{H}}-U_{b}^{r}\right) \lambda_{\mathrm{I}},
\end{gathered}
$$

where $\lambda_{\mathrm{I}}=\max \left((1-\mathrm{q}) \Lambda, \lambda_{m}^{H}\right)$

- If $\lambda_{m}^{H}+\sqrt{c / \alpha^{H}} \leq \mu_{b}$, the decisions and revenue under operational strategy I:

$$
\begin{gathered}
p_{\mathrm{I}}=V_{b}+\alpha^{H}\left(\mu_{b}-\lambda_{\mathrm{I}}\right)+\delta \lambda_{1}-2 \sqrt{c \alpha^{H}}-U_{b}^{r}, \mu_{\mathrm{I}}=\lambda_{\mathrm{I}}+\sqrt{c / \alpha^{H}}, \\
R_{\mathrm{I}}=\left(V_{b}+\alpha^{H}\left(\mu_{b}-\lambda_{1}\right)+\delta \lambda_{1}-2 \sqrt{c \alpha^{H}}-U_{b}^{r}\right) \lambda_{1},
\end{gathered}
$$

where $\lambda_{\mathrm{I}}=\min \left(\lambda_{m}^{H}, \mathrm{q} \Lambda\right)$;

- The decisions and revenue under operational strategy II:

$$
\begin{gathered}
p_{\mathrm{II}}=V_{b}+\alpha^{L}\left(\mu_{b}-\lambda_{\mathrm{II}}\right)+\lambda_{\mathrm{II}}-2 \sqrt{c \alpha^{L}}-U_{b}^{r}, \mu_{\mathrm{II}}=\lambda_{\mathrm{II}}+\sqrt{c / \alpha^{L}}, \\
R_{\mathrm{II}}=\left(V_{b}+\alpha^{L} \mu_{b}+\lambda_{\mathrm{II}}\left(\delta-\alpha^{L}\right)-2 \sqrt{c \alpha^{L}}-U_{b}^{r}\right) \lambda_{\mathrm{II}}
\end{gathered}
$$

where $\lambda_{\mathrm{II}}=\max \left((1-q) \Lambda, \min \left(\Lambda, \mu_{b}-\sqrt{c / \alpha^{L}}\right)\right)$.

In equilibrium, based on the above two strategies, the service provider's optimal decisions, the market coverage and the revenue are given by the following result (the proof is in the appendix):

Proposition 4. Under the case $\alpha^{L}<\delta<\alpha^{H}$, depending on the comparison between $\mu_{b}$ and $\lambda_{m}^{H}+\sqrt{c / \alpha^{H}}$, the following cases hold:

- For the case $\lambda_{m}^{H}+\sqrt{c / \alpha^{H}}>\mu_{b}$, the optimal price and service rate decisions depend on the comparison of revenues from the two strategies.

- If $R_{\mathrm{I}} \geq R_{\mathrm{II}}$, then $\lambda^{*}=\max \left((1-\mathrm{q}) \Lambda, \lambda_{m}^{H}\right)$, and $\mu^{*}=\mu_{\mathrm{I}}, p^{*}=p_{\mathrm{I}}, R^{*}=R_{\mathrm{I}}$;

- If $R_{\mathrm{I}}<R_{\mathrm{II}}$, then $\lambda^{*}=\max \left((1-q) \Lambda, \min \left(\Lambda, \mu_{b}-\sqrt{c / \alpha^{L}}\right)\right)$, and $\mu^{*}=\mu_{\mathrm{II}}, p^{*}=p_{\mathrm{II}}$, $R^{*}=R_{\mathrm{II}}$.

- For the case $\lambda_{m}^{H}+\sqrt{c / \alpha^{H}} \leq \mu_{b}$, the optimal price and service rate decisions depend on the comparison of the revenues from the two strategies.

- If $R_{\mathrm{I}} \geq R_{\mathrm{II}}$, then $\lambda^{*}=\min \left(\lambda_{m}^{H}, q \Lambda\right)$, and $\mu^{*}=\mu_{\mathrm{I}}, p^{*}=p_{\mathrm{I}}, R^{*}=R_{\mathrm{I}}$;

- If $R_{\mathrm{I}}<R_{\mathrm{II}}$, then $\lambda^{*}=\max \left((1-q) \Lambda, \min \left(\Lambda, \mu_{b}-\sqrt{c / \alpha^{L}}\right)\right)$, and $\mu^{*}=\mu_{\mathrm{II}}, p^{*}=p_{\mathrm{II}}$, $R^{*}=R_{\mathrm{II}}$.

By Proposition 4, the proportions of high sensitive customers and low sensitive customers, and the base service rate $\mu_{b}$, play important roles in the optimal decisions. It may be optimal for the service provider to fully cover or partially cover the high sensitive customers if the proportion of those customers is large. While under certain situations, the optimal 
decision is to cover or partially cover low sensitive customers. There are also cases that it is optimal to cover a certain portion of high sensitive customers and all the low sensitive customers. Under some cases, the optimal arrival rate is $\mu_{b}-\sqrt{c / \alpha^{L}}$, i.e., the optimal service rate is set at the base service rate level which determines the equilibrium arrival rate, increasing in the customer intensity of the low sensitive customers and decreasing in the waiting cost per unit of time.

(3) Operational decisions with $\delta \leq \alpha^{L}$.

For the case with $\delta \leq \alpha^{L}$, the optimal arrival rates with $\alpha=\alpha^{H}$ and $\alpha=\alpha^{L}$ are denoted respectively:

$$
\lambda_{m}^{H}=\min \left(\frac{V_{b}+\alpha^{H} \mu_{b}-2 \sqrt{c \alpha^{H}}-U_{b}^{r}}{2\left(\alpha^{H}-\delta\right)}, \Lambda\right), \lambda_{m}^{L}=\min \left(\frac{V_{b}+\alpha^{L} \mu_{b}-2 \sqrt{c \alpha^{L}}-U_{b}^{r}}{2\left(\alpha^{L}-\delta\right)}, \Lambda\right) .
$$

The price and service rate decisions under two operational strategies are denoted as below:

- If $\lambda_{m}^{H}+\sqrt{c / \alpha^{H}}>\mu_{b}$, the decisions and revenue under operational strategy I:

$$
\begin{gathered}
p_{\mathrm{I}}=V_{b}+\alpha^{H}\left(\mu_{b}-\lambda_{\mathrm{I}}\right)+\delta \lambda_{\mathrm{I}}-2 \sqrt{c \alpha^{H}}-U_{b}^{r}, \mu_{\mathrm{I}}=\lambda_{\mathrm{I}}+\sqrt{c / \alpha^{H}}, \\
R_{\mathrm{I}}=\left(V_{b}+\alpha^{H}\left(\mu_{b}-\lambda_{\mathrm{I}}\right)+\delta \lambda_{\mathrm{I}}-2 \sqrt{c \alpha^{H}}-U_{b}^{r}\right) \lambda_{\mathrm{I}},
\end{gathered}
$$

where $\lambda_{\mathrm{I}}=\max \left((1-\mathrm{q}) \Lambda, \lambda_{m}^{H}\right)$;

- If $\lambda_{m}^{H}+\sqrt{c / \alpha^{H}} \leq \mu_{b}$, the decisions and revenue under operational strategy I:

$$
\begin{gathered}
p_{\mathrm{I}}=V_{b}+\alpha^{H}\left(\mu_{b}-\lambda_{\mathrm{I}}\right)+\delta \lambda_{1}-2 \sqrt{c \alpha^{H}}-U_{b}^{r}, \mu_{\mathrm{I}}=\lambda_{1}+\sqrt{c / \alpha^{H}}, \\
R_{\mathrm{I}}=\left(V_{b}+\alpha^{H}\left(\mu_{b}-\lambda_{1}\right)+\delta \lambda_{1}-2 \sqrt{c \alpha^{H}}-U_{b}^{r}\right) \lambda_{1},
\end{gathered}
$$

where $\lambda_{1}=\min \left(\lambda_{m}^{H}, \mathrm{q} \Lambda\right)$;

- If $\lambda_{m}^{L}+\sqrt{c / \alpha^{L}}>\mu_{b}$, the decisions and revenue under operational strategy II:

$$
\begin{gathered}
p_{\mathrm{II}}=V_{b}+\alpha^{L}\left(\mu_{b}-\lambda_{\mathrm{II}}\right)+\delta \lambda_{\mathrm{II}}-2 \sqrt{c \alpha^{L}}-U_{b}^{r}, \mu_{\mathrm{II}}=\lambda_{\mathrm{II}}+\sqrt{c / \alpha^{L}}, \\
R_{\mathrm{II}}=\left(V_{b}+\alpha^{L}\left(\mu_{b}-\lambda_{\mathrm{II}}\right)+\delta \lambda_{\mathrm{II}}-2 \sqrt{c \alpha^{L}}-U_{b}^{r}\right) \lambda_{\mathrm{II}},
\end{gathered}
$$

where $\lambda_{\text {II }}=\min \left((1-\mathrm{q}) \Lambda, \lambda_{m}^{L}\right)$;

- If $\lambda_{m}^{L}+\sqrt{c / \alpha^{L}} \leq \mu_{b}$, the decisions and revenue under operational strategy II:

$$
\begin{gathered}
p_{\mathrm{II}}=V_{b}+\alpha^{L}\left(\mu_{b}-\lambda_{\mathrm{II}}\right)+\delta \lambda_{\mathrm{II}}-2 \sqrt{c \alpha^{L}}-U_{b}^{r}, \mu_{\mathrm{II}}=\lambda_{\mathrm{II}}+\sqrt{c / \alpha^{L}}, \\
R_{\mathrm{II}}=\left(V_{b}+\alpha^{L}\left(\mu_{b}-\lambda_{\mathrm{II}}\right)+\delta \lambda_{\mathrm{II}}-2 \sqrt{c \alpha^{L}}-U_{b}^{r}\right) \lambda_{\mathrm{II}},
\end{gathered}
$$

where $\lambda_{\text {II }}=\max \left(\lambda_{m}^{L}, \mathrm{q} \Lambda\right)$.

Then, the following result captures the service provider's optimal decisions, the market coverage and the revenues in equilibrium (the proof is in the appendix): 
Proposition 5. Under the case $\delta \leq \alpha^{L}$, the following cases hold:

- For the case with $\min \left(\lambda_{m}^{H}+\sqrt{c / \alpha^{H}}, \lambda_{m}^{L}+\sqrt{c / \alpha^{L}}\right) \geq \mu_{b}$, the optimal price and service rate decisions depend on the comparison of the revenues from the two strategies.

- If $R_{\mathrm{I}} \geq R_{\mathrm{II}}$, then $\lambda^{*}=\max \left((1-\mathrm{q}) \Lambda, \lambda_{m}^{H}\right)$, and $\mu^{*}=\mu_{\mathrm{I}}, p^{*}=p_{\mathrm{I}}, R^{*}=R_{\mathrm{I}}$;

- If $R_{\mathrm{I}}<R_{\mathrm{II}}$, then $\lambda^{*}=\min \left((1-\mathrm{q}) \Lambda, \lambda_{m}^{L}\right)$, and $\mu^{*}=\mu_{\mathrm{II}}, p^{*}=p_{\mathrm{II}}, R^{*}=R_{\mathrm{II}}$.

- For the case with $\max \left(\lambda_{m}^{H}+\sqrt{c / \alpha^{H}}, \lambda_{m}^{L}+\sqrt{c / \alpha^{L}}\right) \leq \mu_{b}$, the optimal price and service rate decisions depend on the comparison of the revenues from the two strategies.

- If $R_{\mathrm{I}} \geq R_{\mathrm{II}}$, then $\lambda^{*}=\min \left(\lambda_{m}^{H}, \mathrm{q} \Lambda\right)$, and $\mu^{*}=\mu_{\mathrm{I}}, p^{*}=p_{\mathrm{I}}, R^{*}=R_{\mathrm{I}}$;

- If $R_{\mathrm{I}}<R_{\mathrm{II}}$, then $\lambda^{*}=\max \left(\lambda_{m}^{L}, \mathrm{q} \Lambda\right)$, and $\mu^{*}=\mu_{\mathrm{II}}, p^{*}=p_{\mathrm{II}}, R^{*}=R_{\mathrm{II}}$.

- For the case with $\lambda_{m}^{H}+\sqrt{c / \alpha^{H}}<\mu_{b}<\lambda_{m}^{L}+\sqrt{c / \alpha^{L}}$, the optimal price and service rate decisions depend on the comparison of the revenues from the two strategies.

- If $R_{\mathrm{I}} \geq R_{\mathrm{II}}$, then $\lambda^{*}=\min \left(\lambda_{m}^{H}, \mathrm{q} \Lambda\right)$, and $\mu^{*}=\mu_{\mathrm{I}}, p^{*}=p_{\mathrm{I}}, R^{*}=R_{\mathrm{I}}$;

- If $R_{\mathrm{I}}<R_{\mathrm{II}}$, then $\lambda^{*}=\min \left((1-\mathrm{q}) \Lambda, \lambda_{m}^{L}\right)$, and $\mu^{*}=\mu_{\mathrm{II}}, p^{*}=p_{\mathrm{II}}, R^{*}=R_{\mathrm{II}}$.

- For the case with $\lambda_{m}^{L}+\sqrt{c / \alpha^{L}}<\mu_{b}<\lambda_{m}^{H}+\sqrt{c / \alpha^{H}}$, the optimal price and service rate decisions depend on the comparison of revenues between two strategies.

- If $R_{\mathrm{I}} \geq R_{\mathrm{II}}$, then $\lambda^{*}=\max \left((1-\mathrm{q}) \Lambda, \lambda_{m}^{H}\right)$, and $\mu^{*}=\mu_{\mathrm{I}}, p^{*}=p_{\mathrm{I}}, R^{*}=R_{\mathrm{I}}$;

- If $R_{\mathrm{I}}<R_{\mathrm{II}}$, then $\lambda^{*}=\max \left(\lambda_{m}^{L}, \mathrm{q} \Lambda\right)$, and $\mu^{*}=\mu_{\mathrm{II}}, p^{*}=p_{\mathrm{II}}, R^{*}=R_{\mathrm{II}}$.

By Proposition 5, the proportions of the two types of customers, and the base service rate $\mu_{b}$, play important roles in the optimal decisions. Similar to Proposions 3 and 4 , it may be optimal for the service provider to fully cover or partially cover the high sensitive customers if the proportion of these customers is large. While under certain situations, the best decision is to cover or partially cover low sensitive customers. There are also some cases where it is optimal to cover some of the high sensitive customers and all the low sensitive customers. Under some condition, covering a certain portion of low sensitive customers and all the high sensitive customers will be the optimal operational strategy.

To sum up, it can be found that a customer-intensive service provider needs to consider various situations when taking social interactions into consideration. The core of the analysis lies in the comparison among customer intensities $\alpha^{H}, \alpha^{L}$, and the social interaction intensity $\delta$. The service provider could select to fully cover or partially cover the two categories of customers according to their proportions and the relative maginitudes among $\alpha^{H}, \alpha^{L}$, and $\delta$. It has been concluded that the customer-intensive service provider gets more strategic options by making comparison among $\alpha^{H}, \alpha^{L}$, and $\delta$, which is a effective means to avoid potential loss of revenues.

\section{Conclusions}

This paper investigates the price and service rate decisions for a service provider which offers a customer-intensive service to customers under social interactions, where more demand of the service increases customers' perceived service value (which is a key component of 
customers' value perception). Due to the nature of customer-intensive services, customers perceive a low service quality thus a low value if the service speed is high. The quality-speed conundrum requires the service provider to use a low service rate. However, a low service rate constrains the capability of the service provider where customers may have to wait for a long time in the queue, which may reduce the demand of the service. Consequently, the impact of social interactions in customers' value perception is weakened due to a low demand. For the service provider, how to manage price and capacity decisions under the influence of social interactions is critical for the firm to expand the market size and increase revenue by leveraging on the influence of social interactions. This paper contributes to the literature by taking the influence of social interactions into decision-making besides the typical qualityspeed conundrum in the operations of the customer-intensive service.

Social interactions can be beneficial to the customer-intensive service provider as customers' perceived value increases in the size of the served demand under which a higher price can be charged or a higher service speed can be used or both. Potential revenues will be lost without considering the influence of social interactions in customers' value perception. Particularly, if the influence of social interactions is high, the firm can cover the full market, by using a higher service rate and charging a higher price, which lead to a higher expected revenue. This paper also considers the cases with heterogeneous customers, where they have different levels of customer intensity in value perception. The operations of the customerintensive service become more complex when social interactions are present. One key finding from our analysis is that the proportions of high sensitive and low sensitive customers play an important role in the service provider's operational decision-making. It is found that in some cases, it could be optimal for the service provider to cover a certain portion of high sensitive customers and all the low sensitive customers; while under some other conditions, covering a certain portion of low sensitive customers and all the high sensitive customers would be optimal. Thus, for customer-intensive service providers, by taking customers' heterogeneity into consideration, better operational decisions can be made to achieve profit optimization. Furthermore, our findings provide better understandings on the interplay between customers' quality perception and the influence of social interactions in customer-intensive services.

The limitation of the research is that customers are assumed to have the same reservation value when making purchase decisions for ease of analysis. In fact, customers always hold different reservation values because of private preferences. Extending the model to the case with heterogeneous customers in terms of their reservation values would be adopted in future work. A promising direction can be extended for future research is to investigate the operational decisions in a competitive market, where two or several service providers of the same type of or substitutable customer-intensive services compete against each other for the market with consumers whose purchase behaviors are influenced by social interactions.

\section{Funding}

This work was supported by the $<$ National Natural Science Foundation of China $>$ under Grant [number 71502044]; <China Postdoctoral Science Foundation> under Grant [number 2015M570300]. 


\section{Author contributions}

Cheng Zhang LI wrote the first draft of the article, Minghui JIANG and Xuchuan YUAN raised the idea and conceived the study.

\section{References}

Anand, K. S., Paç, M. F., \& Veeraraghavan, S. (2011). Quality-speed conundrum: trade- offs in customer-intensive services. Management Science, 57(1), 40-56. https://doi.org/10.1287/mnsc.1100.1250

Ata, B., \& Shneorson, S. (2006). Dynamic control of an M/M/1 service system with adjustable arrival and service rates. Management Science, 52(11), 1778-1791. https://doi.org/10.1287/mnsc.1060.0587

Bohlmann, R. N., Rosa, J. A., Bolton, R. N., \& Qualls, W. J. (2006). The effect of group interactions on satisfaction judgment: satisfaction escalation. Marketing Science, 25(4), 301-321. https://doi.org/10.1287/mksc.1050.0182

Brock, W. A., \& Durlauf, S. N. (2001). Discrete choice with social interactions. Review of Economic Studies, 68(2), 235-260. https://doi.org/10.1111/1467-937X.00168

Brock, W. A., \& Durlauf, S. N. (2007). Identification of binary choice models with social interactions. Journal of Economics, 140(1), 52-75. https://doi.org/10.1016/j.jeconom.2006.09.002

Campbell, A. (2015). Word of mouth models for sales. Economics Letters, 133, 45-50. https://doi.org/10.1016/j.econlet.2015.04.019

Li, C., Jiang, M., \& Yuan, X. (2018). Managing operations in customer-intensive services with forwardlooking customers. Kybernetes, 47(10), 1941-1955. https://doi.org/10.1108/K-11-2017-0436

Godes, D. (2016). Product policy in markets with word-of-mouth communication. Management Science, 63(1), 267-278. https://doi.org/10.1287/mnsc.2015.2330

Ni, G., Xu, Y., \& Dong, Y. (2013). Price and speed decisions in customer-intensive services with two classes of customers. European Journal of Operational Research, 228(2), 427-436. https://doi.org/10.1016/j.ejor.2013.01.053

Hanson, W. A., \& Puter, D. S. (1996). Hits and misses: herb behavior and online product popularity. Marketing Letters, 7(4), 297-305. https://doi.org/10.1007/BF00435537

Hartmann, W. R. (2010). Demand estimation with social interactions and the implications for targeted marketing. Marketing Science, 29(4), 585-601. https://doi.org/10.1287/mksc.1100.0559

Hassin, R., \& Haviv, M. (2003). To queue or not to queue: equilibrium behavior in queuing systems (1st ed.). Norwell: Kluwer Academic Publishers. https://doi.org/10.1007/978-1-4615-0359-0

Kostami, V., Kostamis, D., \& Ziya, S. (2017). Pricing and capacity allocation for shared services. Manufacturing and Service Operations Management, 19(2), 230-245. https://doi.org/10.1287/msom.2016.0606

Marand, A. J., Tang, O., \& Li, H. (2019). Quandary of service logistics: fast or reliable? European Journal of Operational Research, 275(3), 983-996. https://doi.org/10.1016/j.ejor.2018.12.007

Moretti, E. (2011). Social learning and peer effects in consumption: evidence from movie sales. Review of Economic Studies, 78(1), 356-393. https://doi.org/10.1093/restud/rdq014

Nie, P. Y., Wang, C., \& Yang, Y. C. (2019). Vertical integration maintenance commitments. Journal of Retailing and Consumer Services, 47, 11-16. https://doi.org/10.1016/j.jretconser.2018.10.008

Onnela, J-P., Reed-Tsochas, F., \& Stanley, H. E. (2010). Spontaneous emergence of social influence in online system. Proceedings of the National Academy of Sciences of the United States of America, 107(43), 18375-18380. https://doi.org/10.1073/pnas.0914572107 
Papanastasiou, Y., \& Savva, N. (2017). Dynamic pricing in the presence of social learning and strategic consumers. Management Science, 63(4), 919-939. https://doi.org/10.1287/mnsc.2015.2378

Qiu, L., \& Whinston, A. B. (2017). Operational strategies under behavioral observational learning in social networks. Production and Operations Management, 26(7), 1249-1267. https://doi.org/10.1111/poms.12693

Simonsohn, U., \& Ariely, D. (2008). When rational sellers face nonrational buyers: evidence from herding on eBay. Management Science, 54(9), 1624-1637. https://doi.org/10.1287/mnsc.1080.0881

Spathis, C., Petridou, E., \& Glaveli, N. (2004). Managing service quality in banks: customers' gender effects. Managing Service Quality: An International Journal, 14(1), 90-102. https://doi.org/10.1108/09604520410513695

Stidham, S. (2002). Analysis, design, and control of queueing systems. Operations Research, 50(1), 197216. https://doi.org/10.1287/opre.50.1.197.17783

Veeraraghavan, S., \& Debo, L. (2009). Joining longer queues: information externalities in queue choice. Manufacturing and Service Operations Management, 11(4), 543-562. https://doi.org/10.1287/msom.1080.0239

Li, X., Guo, P., \& Lian, Z. (2016). Quality-speed competition in customer-intensive services with boundedly rational customers. Production and Operations Management, 25(11), 1885-1901. https://doi.org/10.1111/poms.12583

Li, X., Li, Q., Guo, P., \& Lian, Z. (2017). On the uniqueness and stability of equilibrium in qualityspeed competition with boundedly-rational customers: The case with general reward function and multiple servers. International Journal of Production Economics, 193, 726-736. https://doi.org/10.1016/j.ijpe.2017.08.026

Yu, M., Debo, L., \& Kapuscinski, R. (2016). Strategic waiting for consumer-generated quality information: dynamic pricing of new experience goods. Management Science, 62(2), 410-435. https://doi.org/10.1287/mnsc.2014.2134

Yuan, X., \& Hwarng, H. B. (2018). Managing a service system under the influence of social interactions. Working paper. Singapore University of Social Sciences.

Zhang, J., Liu, Y., \& Chen, Y. (2015). Social learning in networks of friends versus strangers. Marketing Science, 34(4), 573-589. https://doi.org/10.1287/mksc.2015.0902 